\title{
Tectonic Settings and Petrogenesis of Pan-African S-Type Granitoids in Anka Schist Belt, NW, Nigeria
}

\author{
Agunleti, Y. S* Najime. T. \\ Department of Geology, Ahmadu Bello University, Zaria. Nigeria
}

\begin{abstract}
Research into geotectonic settings of the Anka schist belt was regionally carried out by (Holt, 1982) stating that there is no evidence of significant partial melting or metasomatism. This research uses chemical data of some PanAfrican granitoids within the Anka schist belt to explain the tectonic setting and petrogenesis of the S-type granitoids intruding the metasediments within the Anka schist belt. Twenty-five (25) representative samples of granitoids was analyzed for major oxides and trace elements composition. Results obtained from the chemical data was used to plot various classification diagrams. The granitoids in the study area plots within the orogenic class and the use of trace element discrimination diagrams as a means of fingerprinting the tectonic environments revealed that the granitoids ranges from syn-collision to late orogenic field. The granitoids which plotted in the syn-collision field is a characteristic of chemical affinity of syn-collision peraluminous granites with highly fractionated volcanic-arc magmas. The petrogenesis of the S-type granitoids in the study area are greywacke sourced which is largely metagreywacke and metapelitic sourced during partial melting.
\end{abstract}

Keywords: Granitoids, Pan-African, S-type, Metasediments, Partial melting

DOI: $10.7176 / \mathrm{JNSR} / 10-2-08$

Publication date: January $31^{\text {st }} 2020$

\section{Introduction}

A summary of work on the Birnin Gwari, Maru and Anka belts was carried out by Ajibade (1980), Egbuniwe (1982) and Holt (1982) respectively and presented in (Fitches et. al., 1987). This summary provides data on sedimentation and tectonics; chemical aspects of volcanic rocks and minor intrusions intimately associated with the metasedimentary rocks; chemical characteristics of the Pan-African plutons cutting the schist belts. They also question the ages of events and geotectonic settings of the schist belts. According (Holt, 1982), all 15 intrusions studied in part of the Anka schist belt are high level plutons emplaced by stoping and diapiric processes such that contacts with host rocks are very rarely exposed but where seen are sharp and discordant, marginal zones containing rafts, screens and angular xenoliths of the host rock and characteristically the plutons have narrow thermal aureoles with assemblages of muscovite, biotite, andalusite, sillimanite, cordierite and garnet in various combinations. Holt (1982) also stated that there is no evidence of significant partial melting or metasomatism at such contacts and that there are no marginal migmatites except of brittle agmatite type with no indications that any of the bodies formed in situ. He concluded that these plutons are products of magmagenesis at levels substantially below the present exposure level. Meanwhile chemical data from the Bunkasau dykes and microtonalites lie close to the calc-alkaline trend on the AFM diagram and have arc and syn-collisional characteristics on discrimination diagrams (Holt, 1982). These dykes were described to have rare-earth and other chemical characteristics similar to those described by (McCurry, 1971) with High-K, calc-alkaline late-tectonic volcanic rocks of intra-continental collision belts. From the work of (Holt, 1982), the only pluton known definitely to precede the main deformation is the Gusau granite gneiss 
that separates the Maru Belt from the Gusau Migmatites of the basement. Chemically, he stated that the plutons individually and collectively follow a calc-alkaline trend on an AFM diagram and excepting the quartz syenites, are calc-alkaline to alkaline on an alkalinity index diagram and that all the plutons fall within the I-type characteristics. Emmermann et al (1975) and Obiora and Ukaegbu (2010) indicated the relevance of the use of trace and rare earth elements variations and their distribution patterns to be useful in tracing the evolution of granitic and basaltic rocks, much that information on magmatic differentiation and on the degree of fractionation can be derived. This study concentrated on the use of major oxide, trace elements and rare earth elements geochemistry to determine the magmatic evolution and petrogenetic characteristics the granitoids in the part of Anka schist belt.

\section{Geological Settings}

Nigeria lies in the late Proterozoic-early Phanerozoic terrain which separates the West African and Congo cratons (Figure 1) (Wright, 1985; Fitches et. al., 1987). Three (3) major litho-petrological components make up the geology of Nigeria (1. Basement Complex which is Pan-African and older (Precambrian) $\geq \pm 600$ million years, 2. Younger Granites which is Jurassic 200-145 million years and 3. Sedimentary Basins which is Cretaceous to Recent $\leq 145$ million years). The basement complex is one of the three major litho-petrological components that make up the geology of Nigeria. The basement complex is made up of the Migmatite-Gneiss Complex, the Older Metasediments and Younger Metasediments, the Older Granites and the Undeformed basic and acidic dykes (Rahaman, 1976). These rocks are intruded by the Younger Granites that comprise several ring complexes centered in North Central Nigeria centred on Jos (Bowden \& Turner, 1974). The Sedimentary Basins, containing sediments of Cretaceous to Tertiary ages, comprise of the Niger Delta, the Anambra Basin, the Benue Trough (Lower, Middle and Upper), the Chad Basin, the Sokoto Basin, the Mid-Niger (Nupe/Bida) Basin and the Dahomey Embayment. According to (Dada, 2006) the Nigerian basement rocks was affected by the 600Ma Pan-African orogeny and it occupies the reactivated region which resulted from plate collision between the passive continental margin of the West African craton and the active Pharusian continental margin.

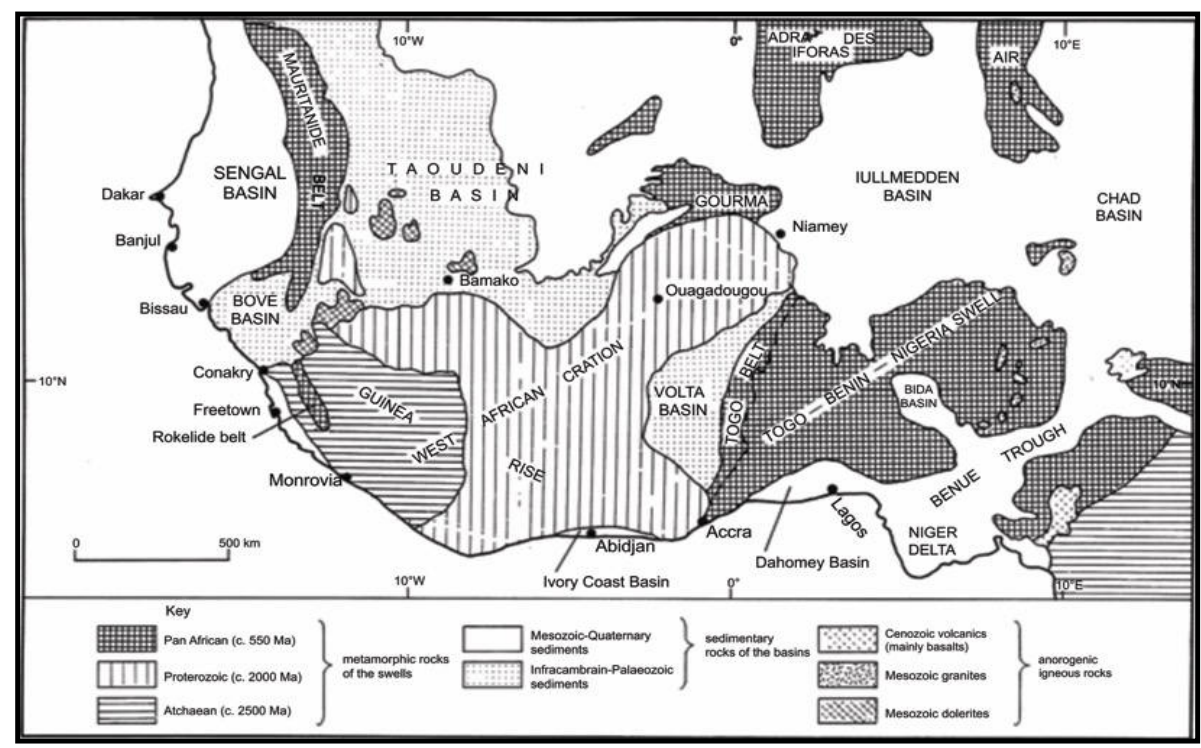

Figure 1: Generalized geological map of Nigeria within the framework of the geology of West Africa (Wright, 1985). 


\subsection{The Anka Schist Belt}

The Anka schist belt lies west of Maru schist belt (Figure 2), the two being separated by the Pan-African Maiinchi granodiorite and by a probably older gabbro-granite- Anka pegmatite complex (APC). The rocks contrast with those of the Maru schist belt and include meta-conglomerates, sandstones, slates, phyllites and acid volcanic rocks (Holt, 1982). Meta-conglomerates formed several units with thicknesses reaching 150 - 250m, but which die out laterally and are interbedded with altered meta-sandstones. They contain rounded to angular boulders and pebbles composed of granite, quartzite, quartz, phyllite and volcanic rocks. In the western part of the belt, phyllites are dominant with some meta-siltstones and meta-sandstones, and rhyolitic to dacitic volcanic rocks. In a partly faulted outlier near Tsado, east of the main belt, green and purple grits are interbedded with shales and siltstones which show fine cross bedding and ripple marks (Turner, 1983). The coarse clastic sediments were deposited in a much higher energy environment than the pelites and iron formation of the Maru Schist Belt and belong to a more active tectonic setting. The laminated and ripple-marked sediments in the east are shallow-water deposits; purple colouring suggests drying and oxidation. A possible environment is an intermontane or rifted basin.

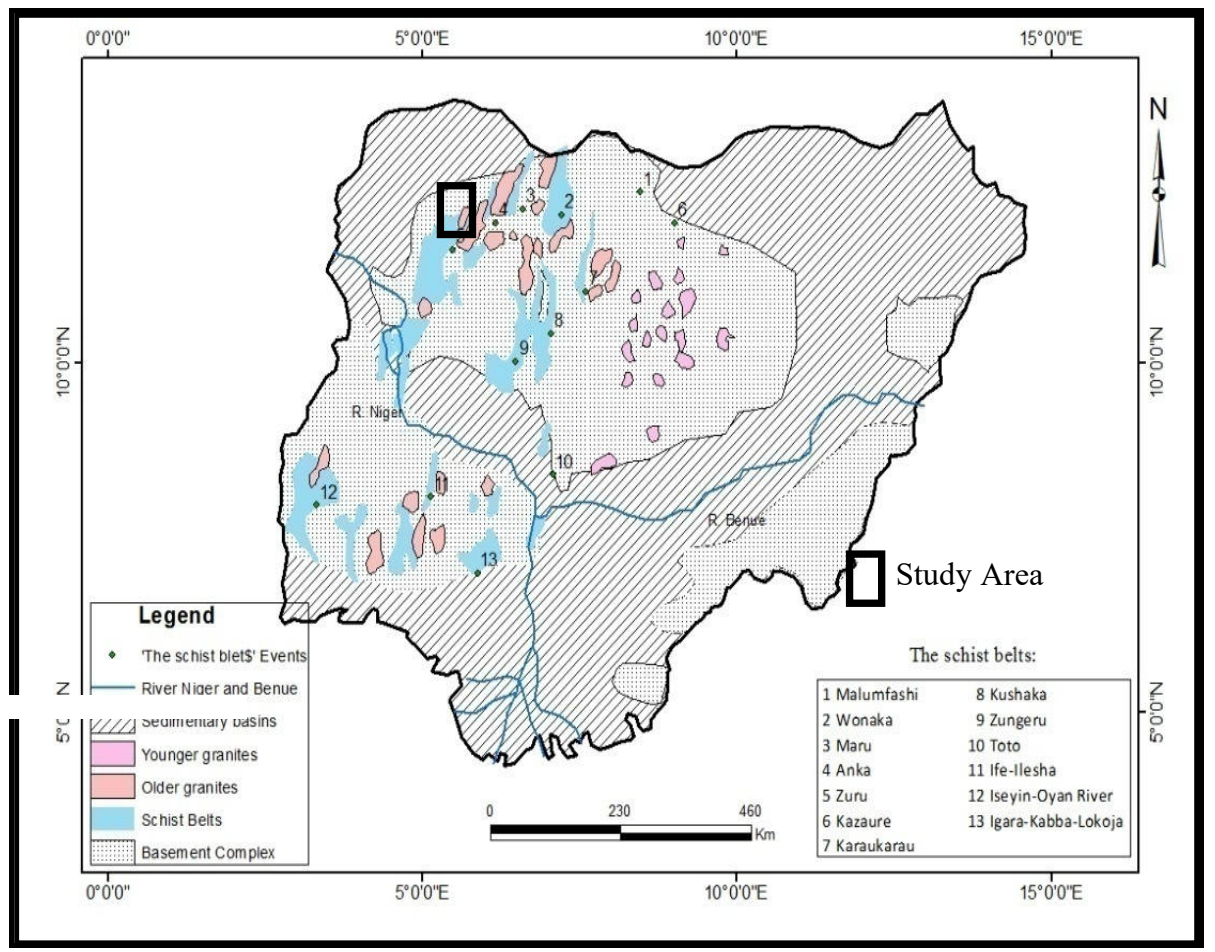

Figure 2: Schist belts localities within the context of the geology of Nigeria after (Woakes et. al., 1987)

\subsection{The Pan-African Granitoids}

Dada (2006) was of the opinion that the term "Pan African Granitoids" be used for the Older Granites not only on the merit of age which was not available at the time they were named Older Granites, but because it covers several important petrologic groups formed at the same time. In Nigeria, the Older Granites are believed to be pre-, synand post- tectonic rocks which cut both the Migmatite-Gneiss-Quartzite complex and the Schist belts. The rocks 
of this suite ranges in composition from tonalites and diorites through granodiorites to true granites and syenites. According to (Jones and Hockey, 1964) there are three main groups of granites, an early phase comprising of granodiorite and quartz diorite, a main phase consisting of coarse porphyritic hornblende and syenite and lastly coarse porphyritic biotite granite. These are followed by a late phase comprising homogenous granite, pegmatite dykes and aplites. The early and late phases are widely distributed. In northern Nigeria, the abundance of PanAfrican granites appears to increase eastward and in the area west of Zaria these occur as isolated intrusions (McCurry, 1973), whereas in the region between Rahama and the Mesozoic-Cenozoic cover the intrusive granites and related rocks envelope remnants of migmatites. McCurry (1973) working mainly west of Zaria divided the granites into two main groups according to their field relationships. The first "syntectonic" group comprised elongate batholithic sheets that are partly concordant, and foliated. The second group "late tectonic" are made up of poorly foliated discordant bodies, rich in mafic xenoliths and having a lower proportion of potash feldspar. The late granites are considered to be the products of widespread mobilization and reactivation of older basement rocks during the Pan-African orogeny. The Older Granites occur intricately associated with the Migmatite-Gneiss Complex and the Schist belts into which they generally intruded. Older Granite rocks occur in most places where rocks of the Migmatite-Gneiss Complex or of the Schist Belt occur. However, Older Granites are particularly noteworthy in and around Wusasa (Zaria), Abuja, Bauchi, Akwanga, Ado-Ekiti and Obudu areas. In Bauchi area and some parts of southwestern Nigeria, most of the Older Granite rocks occur as dark, greenish-grey granites with significant quantities of olivine (fayalite) and pyroxene occurring with quartz, feldspars and micas. For this unusual composition, the Older Granites in these areas are termed Bauchite (in Bauchi area) but initially Oyawoyite (After Professor Oyawoye who first mapped them) in southwestern Nigeria. For uniformity of terminology, both the Bauchites and Oyawoyites constitute the charnockitic rocks (Charnockites) of the Basement Complex. Rahaman (1981) stated that the Older Granites are generally high level intrusions and anataxis has played an important role with suites notable for its general lack of associated mineralization, although the thermal effects may play a role in the remobilization of mineralizing fluids. Geochronologically, whole rock $\mathrm{Rb} / \mathrm{Sr}$ and $\mathrm{U} / \mathrm{Pb}$ studies show that dates on the Granites range from 677 to $518 \mathrm{Ma}$ (Rahaman, 1988). They represent a varied and long lasting (750-450 Ma) magmatic cycle associated with the Pan-African orogeny. The Older Charnockites form an important rock group emplaced during this period. Rahaman (1988) also stated that the Older Granites are the most obvious manifestation of the Pan-African orogeny and represent significant additions of materials (up to $70 \%$ in some places) to the crust, such that an attempt to classify the Older Granites with respect to timing during an orogenic event are valid over only short distances. Contact features between members of the Older Granites suites suggest the coexistence of several magmas and compositionally, the granites plot in the field of calc-alkaline rocks on the AFM diagram (Rahaman, 1988). He also stated that the granites contained significant amount of alkalis that are often slightly corundum normative.

\section{Analytical Methods}

Fifteen (15) representative samples and twenty-five (25) core drill sampled at 5m intervals from the 3 drill holes was analyzed for major oxides and trace elements composition. The samples were crushed and grounded to -150 mesh using a stainless steel grinding jar in Retsch PM400 Ball Mill at Multi-User Science Research Laboratory

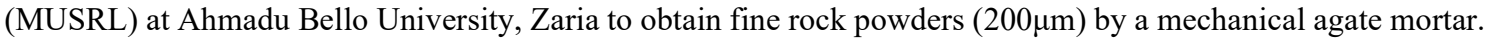


The powdered samples were then shipped to Activation Laboratories (ACTLAB), Ancaster, Ontario, Canada where Inductively Coupled Plasma-Mass Spectrometry (ICP-MS) analytical techniques was used for analysis. A fused beads of the samples are produced by mixing a flux with a sample that has been finely powdered and then heating this mixture to temperatures of $1000^{\circ} \mathrm{C}$ to $1200^{\circ} \mathrm{C}$ in a platinum crucible. This results in the sample being dissolved in the flux, and it is then cast into a mold. The Fused sample is diluted and analyzed by Perkin Elmer Sciex ELAN 9000 ICP-MS instrument to measure the major and trace elements. The information collected from the data system was compared with standard curves. Loss-on-ignition test (LOI), mostly considered to be water and $\mathrm{CO}_{2}$ was measured as the amount of volatiles lost, when the sample is ignited. From $0.9 \mathrm{~g}$ to $1.6 \mathrm{~g}$, each sample was accurately weighed, heated to $1000^{\circ} \mathrm{C}$ and weighed again to calculate the LOI ratio.

\section{Results}

The major oxides, trace and rare elements of the representative rock samples used for this study are presented in Table 1,2 and 3. The classification diagram of $\mathrm{A} / \mathrm{CNK}$ versus $\mathrm{A} / \mathrm{NK}$ (where $\mathrm{A} / \mathrm{CNK}=$ molar $\mathrm{A}_{2} \mathrm{O}_{3} /\left(\mathrm{CaO}+\mathrm{Na}_{2} \mathrm{O}\right.$ $\left.+\mathrm{K}_{2} \mathrm{O}\right)$ and $\mathrm{A} / \mathrm{NK}=$ molar $\mathrm{Al}_{2} \mathrm{O}_{3} /\left(\mathrm{Na}_{2} \mathrm{O}+\mathrm{K}_{2} \mathrm{O}\right)$ (after Shand, 1943) are shown in (Figure 3). The total alkali saturation (TAS) (Cox et al ., 1979; Middlemost, 1985, 1994) are useful tool for classification of igneous rocks and for distinguishing between them are shown in (Figure 4, 5 and 6) while Figure 7 after (De La Roche, et al., 1980). The AFM plot (defined as follows: $\mathrm{A}=(\mathrm{K} 2 \mathrm{O}+\mathrm{Na} 2 \mathrm{O})$ wt. $\% \mathrm{~F}=$ FeOtot wt. $\% \mathrm{M}=\mathrm{MgO}$ wt. $\% \mathrm{~A}+\mathrm{F}+$ $M=100 \%$ ) (Irvine and Baragar, 1971) and binary plots according to (Miyashiro, 1974) are shown in (Figure 8 and 9) respectively. The classification plot after (Peccerillo and Taylor, 1976) for the granitoids are shown in (Figure 10) and binary plots after (Chappell and White, 1974; 1992) to distinguish S-type granitoids from the Itypes are presented in (Figure 11). Tectonic environments of granitoids as categorized by (Maniar and Piccoli, 1989) are 1. island arc granitoids (IAG), 2. continental arc granitoids (CAG), 3. Continental collision granitoids (CCG), 4. Postorogenic granitoids (POG), 5. rift-related granitoids (RRG), 6. Continental epeirogenic uplift granitoids (CEUG) and 7. Oceanic plagiogranites (OP). Of these, IAG, CAG, CCG and POG are considered orogenic granitoids while RRG, CEUG and OP are considered anorogenic granitoids. In this respect, granitoids in the study area plot within the orogenic class (Figure 12). Figure 13 is the tectonic discrimination diagram after (Pearce, et. al., 1984) for granitoids in the study area while Figure 14 clearly show the classification between the granitoids. Roser and Korsch (1986) discrimination plot for the granitoids is shown in (Figure 15) as passive margin and after (Muller, et al., 2001) to indicate a subduction arc-related features from within plate source for the granitoids is presented in (Figure 16). The petrogenesis of the granitoids after (Dahlquist, et al., 2007) are shown in (Figure 17) and after (Altherr, et al., 2000) as shown (Figure 18). 
Table 1: Major oxides contents of rocks and mineral in the study area (\%)

\begin{tabular}{|c|c|c|c|c|c|c|c|c|c|c|c|c|c|c|c|c|c|c|c|c|c|c|c|c|c|c|c|c|}
\hline \multirow{2}{*}{ Oxides } & \multicolumn{3}{|c|}{ Quartz Schist } & \multirow{2}{*}{$\begin{array}{c}\text { Rhyolite } \\
\text { A6 }\end{array}$} & \multicolumn{2}{|c|}{ Syenites } & \multicolumn{3}{|c|}{ Granodiorites } & \multicolumn{5}{|c|}{ Granites (Samples) } & \multicolumn{13}{|c|}{ Granites (Core Samples) } & \multirow{2}{*}{$\begin{array}{c}\text { Malachite-Vein } \\
\text { A74 }\end{array}$} \\
\hline & A4 & A59 & A76a & & A12 & A35 & A13 & A31 & A40 & $\mathrm{A} 76 \mathrm{~b}$ & A11 & $\mathrm{A} 22$ & A28 & $\mathrm{A} 32$ & A98 & $\mathrm{A} 100$ & A104 & A108 & A110 & A114 & A118 & A120 & A102 & A106 & A112 & A116 & A122 & \\
\hline $\mathrm{SiO}_{2}$ & 64.0 & 62.8 & 70.9 & 76.9 & 59.4 & 64.9 & 66.0 & 65.8 & 68.9 & 71.7 & 73.4 & 71.1 & 73.9 & 75.8 & 67.0 & 66.6 & 68.7 & 68.1 & 68.6 & 70.2 & 66.8 & 70.1 & 66.9 & 67.2 & 69.2 & 68.4 & 68.0 & 89.3 \\
\hline $\mathrm{Al}_{2} \mathrm{O}_{3}$ & 12.8 & 15.3 & 14.5 & 12.7 & 18.1 & 16.7 & 15.3 & 14.9 & 14.7 & 15.9 & 14.0 & 14.7 & 14.0 & 12.7 & 15.6 & 14.6 & 13.8 & 15.1 & 17.1 & 14.6 & 14.6 & 14.4 & 15.1 & 13.8 & 14.9 & 15.3 & 15.4 & 1.8 \\
\hline $\mathrm{Fe}_{2} \mathrm{O}_{3}$ & 5.5 & 4.4 & 3.1 & 1.3 & 3.6 & 3.8 & 4.2 & 4.2 & 3.5 & 1.6 & 1.8 & 2.5 & 1.1 & 1.6 & 3.4 & 2.8 & 2.9 & 2.8 & 2.3 & 3.0 & 3.3 & 2.6 & 2.8 & 3.2 & 3.2 & 3.3 & 2.9 & 9 \\
\hline $\mathrm{MnO}$ & 0.1 & 0.0 & 0.0 & 0.1 & 0.1 & 0.1 & 0.1 & 0.1 & 0.1 & 0.0 & 0.0 & 0.1 & 0.0 & 0.1 & 0.0 & 0.1 & 0.0 & 0.0 & 0.0 & 0.0 & 0.1 & 0.0 & 0.0 & 0.1 & 0.0 & 0.0 & .0 & 0.0 \\
\hline $\mathrm{MgO}$ & 2.6 & 1.5 & 0.9 & 0.1 & 1.1 & 0.9 & 2.0 & 2.5 & 0.8 & 0.5 & 0.2 & 0.5 & 0.1 & 0.1 & 0.4 & 0.9 & 0.9 & 0.6 & 0.7 & 0.8 & 0.9 & 0.9 & 0.4 & 0.7 & 1.1 & 1.1 & 1.1 & 0.1 \\
\hline $\mathrm{CaO}$ & 4.2 & 3.4 & 1.1 & 0.1 & 5.3 & 2.4 & 3.0 & 3.7 & 2.2 & 0.5 & 1.2 & 2.1 & 0.9 & 0.6 & 2.4 & 3.1 & 2.2 & 2.5 & 0.8 & 1.6 & 3.2 & 2.2 & 3 & 3.1 & 1.7 & 1.5 & 2.3 & .1 \\
\hline $\mathrm{Na}_{2} \mathrm{O}$ & 2.2 & 2.2 & 3.6 & 4.6 & 3.7 & 4.2 & 3.3 & 3.7 & 3.9 & 5.4 & 3.6 & 3.8 & 3.6 & 4.3 & 4.0 & 4.2 & 3.8 & 4.0 & 4.2 & 4.4 & 4.3 & 4.3 & 3.9 & 3.6 & 4.6 & 3.8 & 4 & .1 \\
\hline $\mathrm{K}_{2} \mathrm{O}$ & 5.8 & 8.1 & 4.6 & 3.9 & 6.7 & 5.0 & 5.0 & 4.0 & 3.7 & 3.3 & 5.2 & 4.5 & 5.3 & 4.5 & 3.9 & 3.3 & 3.8 & 4.0 & 4.3 & 3.6 & 3.2 & 3.0 & 3.7 & 3.7 & 3.3 & 3.9 & 8 & 0 \\
\hline $\mathrm{TiO}_{2}$ & 0.6 & 0.5 & 0.4 & 0.1 & 0.4 & 0.5 & 0.6 & 0.4 & 0.4 & 0.2 & 0.2 & 0.3 & 0.1 & 0.2 & 0.4 & 0.4 & 0.4 & 0.4 & 0.5 & 0.3 & 0.4 & 0.4 & 0.4 & 0.4 & 0.4 & 0.4 & 0.4 & .0 \\
\hline $\mathrm{P}_{2} \mathrm{O}_{5}$ & 0.4 & 0.4 & 0.2 & 0.0 & 0.1 & 0.1 & 0.2 & 0.1 & 0.1 & 0.1 & 0.0 & 0.1 & 0.0 & 0.0 & 0.2 & 0.2 & 0.2 & 0.1 & 0.2 & 0.1 & 0.2 & 0.2 & 0.2 & 0.2 & 0.2 & 0.2 & 0.2 & 0 \\
\hline LOI & 2.2 & 1.5 & 1.3 & 0.3 & 1.3 & 1.0 & 0.8 & 1.2 & 1.3 & 1.0 & 0.4 & 0.5 & 0.6 & 0.7 & 2.9 & 4.1 & 3.3 & 2.8 & 1.9 & 2.0 & 2.9 & 2.5 & 3.8 & 4.0 & 1.9 & 2.6 & 1.7 & .6 \\
\hline Total & 100.4 & 100.1 & 100.6 & 99.8 & 99.9 & 99.6 & 100.3 & 100.6 & 99.6 & 100.1 & 100.1 & 100.1 & 99.5 & 100.6 & 100.2 & 100.2 & 100.0 & 100.6 & 100.4 & 100.7 & 99.8 & 100.6 & 100.4 & 100.1 & 100.5 & 100.3 & 100.1 & 94.4 \\
\hline Ratios & & & & & & & & & & & & $=\mathrm{Al}_{2} \mathrm{O}_{3}$ & & $=\mathrm{CaO}$ & & $\mathrm{I}=\mathrm{Na}_{2} \mathrm{O}$ & & $\mathrm{K}=\mathrm{K}_{2} \mathrm{O}$ & & & & & & & & & & \\
\hline $\mathrm{K}$ & 4.8 & 6.7 & 3.8 & 3.2 & 5.6 & 4.2 & 4.2 & 3.3 & 3.1 & 2.7 & 4.3 & 3.7 & 4.4 & 3.7 & 3.2 & 2.7 & 3.2 & 3.3 & 3.6 & 3.0 & 2.7 & 2.5 & 3.1 & 3.1 & 2.8 & 3.2 & 3.1 & 0.5 \\
\hline CNK & 12.3 & 13.7 & 9.2 & 8.5 & 15.8 & 11.6 & 11.3 & 11.4 & 9.8 & 9.2 & 10.0 & 10.4 & 9.7 & 9.4 & 10.2 & 10.6 & 9.8 & 10.5 & 9.3 & 9.6 & 10.6 & 9.5 & 10.9 & 10.5 & 9.6 & 9.2 & 10.4 & 0.7 \\
\hline $\mathrm{NK}$ & 8.1 & 10.2 & 8.1 & 8.4 & 10.4 & 9.2 & 8.3 & 7.7 & 7.6 & 8.6 & 8.9 & 8.3 & 8.9 & 8.8 & 7.8 & 7.5 & 7.6 & 8.0 & 8.5 & 7.9 & 7.5 & 7.3 & 7.6 & 7.3 & 7.9 & 7.7 & 8.2 & 0.6 \\
\hline $\mathrm{A} / \mathrm{CNK}$ & 1.0 & 1.1 & 1.6 & 1.5 & 1.2 & 1.4 & 1.4 & 1.3 & 1.5 & 1.7 & 1.4 & 1.4 & 1.4 & 1.4 & 1.5 & 1.4 & 1.4 & 1.4 & 1.8 & 1.5 & 1.4 & 1.5 & 1.4 & 1.3 & 1.5 & 1.7 & 1.5 & 2.6 \\
\hline A/NK & 1.6 & 1.5 & 1.8 & 1.5 & 1.7 & 1.8 & 1.8 & 1.9 & 1.9 & 1.8 & 1.6 & 1.8 & 1.6 & 1.4 & 2.0 & 2.0 & 1.8 & 1.9 & 2.0 & 1.8 & 2.0 & 2.0 & 2.0 & 1.9 & 1.9 & 2.0 & 1.9 & 2.8 \\
\hline
\end{tabular}

Table 2: Trace elements contents of rocks and mineral in the study area (ppm)

\begin{tabular}{|c|c|c|c|c|c|c|c|c|c|c|c|c|c|c|c|c|c|c|c|c|c|c|c|c|c|c|c|c|}
\hline \multirow{2}{*}{ Eements } & \multicolumn{3}{|c|}{ Quartz Schist } & \multirow{2}{*}{$\begin{array}{c}\text { Rhyolite } \\
\text { A6 }\end{array}$} & \multicolumn{2}{|c|}{ Syenites } & \multicolumn{3}{|c|}{ Granodiorites } & \multicolumn{5}{|c|}{ Granites (Samples) } & \multicolumn{13}{|c|}{ Granites (Core Samples) } & \multirow{2}{*}{$\begin{array}{c}\text { Malachite-Veir } \\
\text { A74 }\end{array}$} \\
\hline & A4 & A59 & A76a & & A12 & A35 & A13 & A31 & A40 & A76b & A11 & A22 & A28 & A32 & A98 & A100 & A104 & A108 & A110 & A114 & A118 & A120 & A102 & A106 & A112 & A116 & A122 & \\
\hline Se & 7.0 & 5.0 & 4.0 & 2.0 & 5.0 & 5.0 & 8.0 & 6.0 & 5.0 & 6.0 & 1.0 & 3.0 & 1.0 & 2.0 & 5.0 & 4.0 & 4.0 & 4.0 & 5.0 & 3.0 & 4.0 & 4.0 & 5.0 & 5.0 & 5.0 & 4.0 & 4.0 & 1.0 \\
\hline Be & 4.0 & 3.0 & 3.0 & 2.0 & 4.0 & 3.0 & 4.0 & 3.0 & 3.0 & 4.0 & 6.0 & 4.0 & 4.0 & 4.0 & 2.0 & 2.0 & 2.0 & 2.0 & 3.0 & 2.0 & 2.0 & 2.0 & 2.0 & 2.0 & 2.0 & 3.0 & 2.0 & 1.0 \\
\hline $\mathrm{v}$ & 93.0 & 79.0 & 38.0 & 7.0 & 49.0 & 47.0 & 60.0 & 53.0 & 42.0 & 19.0 & 8.0 & 27.0 & 6.0 & 7.0 & 48.0 & 42.0 & 45.0 & 43.0 & 55.0 & 54.0 & 44.0 & 47.0 & 45.0 & 48.0 & 46.0 & 49.0 & 44.0 & 6.0 \\
\hline Sr & 1565.0 & 1848.0 & 650.0 & 21.0 & 763.0 & 468.0 & 429.0 & 750.0 & 561.0 & 713.0 & 241.0 & 404.0 & 194.0 & 41.0 & 557.0 & 801.0 & 586.0 & 605.0 & 338.0 & 495.0 & 876.0 & 492.0 & 652.0 & 574.0 & 659.0 & 542.0 & 746.0 & 39.0 \\
\hline $\mathbf{Y}$ & 14.0 & 14.0 & 8.0 & 24.0 & 41.0 & 33.0 & 18.0 & 10.0 & 28.0 & 2.0 & 9.0 & 16.0 & 9.0 & 28.0 & 9.0 & 8.0 & 7.0 & 8.0 & 7.0 & 6.0 & 8.0 & 7.0 & 8.0 & 9.0 & 8.0 & 6.0 & 9.0 & 2.0 \\
\hline $\mathrm{Zr}$ & 27.0 & 35.0 & 150.0 & 80.0 & 200.0 & 387.0 & 267.0 & 158.0 & 226.0 & 74.0 & 132.0 & 155.0 & 60.0 & 114.0 & 163.0 & 151.0 & 137.0 & 150.0 & 182.0 & 120.0 & 165.0 & 154.0 & 156.0 & 155.0 & 166.0 & 164.0 & 170.0 & 13.0 \\
\hline Ba & 2247.0 & 3047.0 & 1280.0 & 79.0 & 2032.0 & 2008.0 & 1358.0 & 1622.0 & 1932.0 & 905.0 & 961.0 & 1068.0 & 871.0 & 62.0 & 1354.0 & 1115.0 & 1408.0 & 1367.0 & 1313.0 & 1277.0 & 1106.0 & 1084.0 & 1484.0 & 1380.0 & 1125.0 & 1417.0 & 1544.0 & 737.0 \\
\hline $\mathrm{Cr}$ & 150.0 & 0.0 & 30.0 & 37.0 & 0.0 & 30.0 & 70.0 & 30.0 & 30.0 & 20.0 & 0.0 & 30.0 & 30.0 & 30.0 & 30.0 & 30.0 & 30.0 & 30.0 & 30.0 & 30.0 & 30.0 & 30.0 & 30.0 & 30.0 & 30.0 & 30.0 & 30.0 & 20.0 \\
\hline Co & 16.0 & 12.0 & 6.0 & 3.0 & 8.0 & 9.0 & 11.0 & 8.0 & 12.0 & 3.0 & 8.0 & 8.0 & 12.0 & 9.0 & 5.0 & 4.0 & 8.0 & 6.0 & 6.0 & 5.0 & 6.0 & 6.0 & 4.0 & 8.0 & 6.0 & 6.0 & 6.0 & 6.0 \\
\hline $\mathrm{Ni}$ & 70.0 & 65.0 & 20.0 & 45.0 & 18.0 & 20.0 & 30.0 & 30.0 & 30.0 & 20.0 & 20.0 & 20.0 & 30.0 & 20.0 & 30.0 & 30.0 & 30.0 & 30.0 & 20.0 & 20.0 & 30.0 & 25.0 & 20.0 & 20.0 & 20.0 & 20.0 & 20.0 & 20.0 \\
\hline $\mathrm{Cu}$ & 170.0 & 175.0 & 20.0 & 8.0 & 8.0 & 12.0 & 10.0 & 8.0 & 6.0 & 10.0 & 12.0 & 10.0 & 10.0 & 8.0 & 8.0 & 8.0 & 10.0 & 10.0 & 10.0 & 10.0 & 10.0 & 8.0 & 10.0 & 20.0 & 10.0 & 10.0 & 20.0 & 10000.0 \\
\hline Zn & 80.0 & 72.0 & 80.0 & 40.0 & 30.0 & 60.0 & 60.0 & 50.0 & 50.0 & 40.0 & 40.0 & 40.0 & 60.0 & 50.0 & 50.0 & 45.0 & 50.0 & 60.0 & 60.0 & 25.0 & 40.0 & 30.0 & 50.0 & 70.0 & 70.0 & 60.0 & 60.0 & 1380.0 \\
\hline $\mathrm{Ga}$ & 20.0 & 25.0 & 22.0 & 20.0 & 22.0 & 20.0 & 20.0 & 25.0 & 21.0 & 21.0 & 20.0 & 20.0 & 20.0 & 20.0 & 21.0 & 21.0 & 21.0 & 20.0 & 20.0 & 21.0 & 20.0 & 20.0 & 21.0 & 20.0 & 21.0 & 21.0 & 22.0 & 3.0 \\
\hline $\mathrm{Ge}$ & 1.0 & 1.0 & 1.0 & 1.0 & 1.0 & 1.0 & 1.0 & 1.0 & 1.0 & 1.0 & 1.0 & 1.0 & 1.0 & 1.0 & 1.0 & 1.0 & 1.0 & 1.0 & 1.0 & 1.0 & 1.0 & 1.0 & 1.0 & 1.0 & 1.0 & 1.0 & 1.0 & 11.0 \\
\hline As & 5.0 & 3.0 & 6.0 & 5.0 & 6.0 & 5.0 & 5.0 & 5.0 & 5.0 & 5.0 & 6.0 & 5.0 & 5.0 & 6.0 & 5.0 & 5.0 & 5.0 & 6.0 & 5.0 & 5.0 & 5.0 & 5.0 & 5.0 & 6.0 & 5.0 & 5.0 & 5.0 & 2000.0 \\
\hline $\mathbf{R b}$ & 131.0 & 128.0 & 141.0 & 108.0 & 119.0 & 114.0 & 207.0 & 119.0 & 112.0 & 98.0 & 123.0 & 123.0 & 121.0 & 106.0 & 125.0 & 118.0 & 125.0 & 125.0 & 126.0 & 124.0 & 124.0 & 125.0 & 122.0 & 124.0 & 113.0 & 125.0 & 112.0 & 24.0 \\
\hline $\mathrm{Nb}$ & 8.0 & 8.0 & 11.0 & 11.0 & 6.0 & 8.0 & 13.0 & 8.0 & 7.0 & 6.0 & 8.0 & 12.0 & 8.0 & 6.0 & 4.0 & 8.0 & 9.0 & 9.0 & 9.0 & 8.0 & 8.0 & 8.0 & 9.0 & 8.0 & 9.0 & 7.0 & 8.0 & 2.0 \\
\hline Mo & 2.0 & 2.0 & 4.0 & 3.0 & 2.0 & 2.0 & 2.0 & 2.0 & 2.0 & 2.0 & 2.0 & 2.0 & 2.0 & 2.0 & 2.0 & 2.0 & 2.0 & 2.0 & 2.0 & 2.0 & 2.0 & 2.0 & 2.0 & 2.0 & 2.0 & 2.0 & 12.0 & 5.0 \\
\hline Ag & 0.5 & 0.5 & 0.7 & 0.8 & 0.8 & 0.6 & 0.9 & 0.7 & 0.5 & 0.5 & 0.6 & 0.6 & 0.6 & 0.8 & 0.5 & 0.5 & 0.4 & 0.6 & 0.4 & 0.5 & 0.5 & 0.6 & 0.6 & 0.6 & 0.6 & 0.6 & 0.6 & 55.7 \\
\hline In & 0.2 & 0.2 & 0.2 & 0.2 & 0.2 & 0.2 & 0.2 & 0.2 & 0.2 & 0.2 & 0.2 & 0.2 & 0.2 & 0.2 & 0.2 & 0.2 & 0.2 & 0.2 & 0.2 & 0.2 & 0.2 & 0.2 & 0.2 & 0.2 & 0.2 & 0.2 & 0.2 & 0.2 \\
\hline Sn & 2.0 & 2.0 & 1.0 & 1.0 & 2.0 & 1.0 & 3.0 & 1.0 & 1.0 & 1.0 & 1.0 & 1.0 & 1.0 & 1.0 & 1.0 & 1.0 & 1.0 & 1.0 & 1.0 & 1.0 & 1.0 & 1.0 & 1.0 & 1.0 & 1.0 & 1.0 & 1.0 & 1.0 \\
\hline Sb & 2.8 & 3.1 & 33.3 & 8.0 & 18.0 & 16.0 & 0.6 & 16.0 & 22.0 & 7.0 & 14.0 & 25.0 & 22.0 & 19.0 & 23.0 & 19.0 & 22.0 & 20.0 & 21.0 & 22.0 & 23.0 & 23.0 & 25.0 & 14.8 & 6.2 & 19.1 & 5.0 & 200.0 \\
\hline Cs & 0.8 & 0.5 & 3.3 & 2.8 & 3.7 & 4.4 & 5.5 & 2.9 & 2.8 & 1.8 & 0.9 & 3.6 & 5.1 & 4.1 & 2.5 & 3.1 & 3.6 & 4.2 & 4.6 & 2.6 & 3.6 & 2.8 & 3.7 & 3.5 & 4.6 & 4.7 & 3.1 & 0.5 \\
\hline $\mathbf{T a}$ & 0.6 & 0.8 & 1.1 & 0.5 & 0.4 & 2.1 & 1.3 & 0.7 & 0.5 & 0.7 & 0.8 & 1.6 & 0.7 & 0.4 & 0.7 & 0.4 & 0.3 & 0.3 & 0.9 & 0.3 & 0.7 & 0.4 & 0.7 & 0.8 & 0.7 & 0.6 & 0.8 & 0.1 \\
\hline w & 1.0 & 1.0 & 1.0 & 1.0 & 1.0 & 1.0 & 1.0 & 1.0 & 1.0 & 1.0 & 1.0 & 1.0 & 1.0 & 1.0 & 1.0 & 1.0 & 1.0 & 1.0 & 1.0 & 1.0 & 1.0 & 1.0 & 1.0 & 1.0 & 3.0 & 1.0 & 1.0 & 1.0 \\
\hline $\mathrm{TI}$ & 0.6 & 0.5 & 0.6 & 0.4 & 0.5 & 0.8 & 0.8 & 0.8 & 0.8 & 0.4 & 0.4 & 0.5 & 0.7 & 0.8 & 0.8 & 0.5 & 0.6 & 0.4 & 0.5 & 0.7 & 0.5 & 0.6 & 0.5 & 0.6 & 0.5 & 0.5 & 0.5 & 1.0 \\
\hline $\mathbf{P b}$ & 33.0 & 25.0 & 40.0 & 25.0 & 21.0 & 12.0 & 33.0 & 23.0 & 16.0 & 27.0 & 22.0 & 32.0 & 28.0 & 25.0 & 17.0 & 22.0 & 16.0 & 18.0 & 18.0 & 21.0 & 19.0 & 18.0 & 19.0 & 38.0 & 20.0 & 41.0 & 24.0 & 10000.0 \\
\hline $\mathbf{B i}$ & 0.4 & 0.4 & 0.4 & 0.4 & 0.4 & 0.4 & 0.4 & 0.4 & 0.4 & 0.4 & 0.4 & 0.4 & 0.4 & 0.4 & 0.4 & 0.4 & 0.4 & 0.4 & 0.4 & 0.4 & 0.4 & 0.4 & 0.4 & 0.4 & 0.4 & 0.4 & 0.4 & 7.1 \\
\hline Th & 26.3 & 24.0 & 13.2 & 19.5 & 22.4 & 16.8 & 30.2 & 18.5 & 12.6 & 6.6 & 18.4 & 22.5 & 14.7 & 14.7 & 15.9 & 15.4 & 20.3 & 20.5 & 21.8 & 21.7 & 15.7 & 16.2 & 15.1 & 18.1 & 16.6 & 11.6 & 20.5 & 2.2 \\
\hline $\mathbf{U}$ & 4.0 & 3.0 & 3.7 & 2.6 & 3.6 & 1.2 & 8.4 & 1.8 & 1.1 & 1.6 & 1.9 & 2.0 & 2.0 & 1.6 & 2.1 & 2.5 & 1.9 & 1.2 & 1.3 & 1.4 & 1.3 & 1.5 & 1.6 & 2.0 & 2.3 & 1.3 & 2.3 & 20.5 \\
\hline
\end{tabular}


Table 3: Rare Earth Elements (REE) contents (ppm) of rocks and mineral in the study area

\begin{tabular}{|c|c|c|c|c|c|c|c|c|c|c|c|}
\hline \multirow[b]{2}{*}{ S ample } & \multicolumn{2}{|c|}{ Quartz Schist } & \multirow{2}{*}{$\begin{array}{c}\text { Granite } \\
\text { A76b } \\
\end{array}$} & \multirow{2}{*}{$\begin{array}{c}\text { Granodiorite } \\
\text { A13 } \\
\end{array}$} & \multicolumn{5}{|c|}{ Core Samples (Granites) } & \multirow{2}{*}{$\begin{array}{c}\text { Malachite-Vein } \\
\text { A74 } \\
\end{array}$} & \multirow{2}{*}{$\Sigma$} \\
\hline & A4 & A76a & & & A102 & A106 & A112 & A116 & A122 & & \\
\hline $\mathbf{C e}$ & 384.0 & 80.3 & 29.2 & 92.2 & 92.7 & 107.0 & 85.7 & 63.1 & 105.0 & 11.6 & 1050.8 \\
\hline Pr & 40.4 & 8.8 & 2.5 & 10.2 & 9.3 & 10.6 & 8.7 & 6.7 & 10.7 & 1.1 & 109.0 \\
\hline Nd & 128.0 & 30.2 & 8.9 & 36.8 & 32.5 & 36.3 & 30.0 & 22.9 & 36.8 & 3.6 & 366.0 \\
\hline S m & 15.6 & 4.6 & 1.4 & 6.3 & 4.8 & 5.4 & 4.6 & 3.5 & 5.4 & 0.5 & 52.1 \\
\hline Eu & 3.6 & 1.1 & 0.4 & 1.4 & 1.1 & 1.3 & 1.1 & 0.8 & 1.3 & 0.1 & 12.1 \\
\hline Gd & 7.8 & 2.7 & 0.9 & 4.6 & 2.8 & 3.2 & 2.8 & 2.2 & 3.2 & 0.3 & 30.5 \\
\hline $\mathbf{T b}$ & 0.8 & 0.4 & 0.1 & 0.6 & 0.3 & 0.4 & 0.3 & 0.3 & 0.4 & 0.1 & 3.7 \\
\hline Ho & 0.5 & 0.3 & 0.1 & 0.6 & 0.3 & 0.3 & 0.3 & 0.3 & 0.3 & 0.1 & 3.1 \\
\hline $\mathbf{E r}$ & 1.3 & 0.9 & 0.3 & 1.8 & 0.8 & 0.9 & 0.8 & 0.7 & 0.9 & 0.1 & 8.5 \\
\hline $\mathbf{T m}$ & 0.2 & 0.1 & 0.1 & 0.3 & 0.1 & 0.1 & 0.1 & 0.1 & 0.1 & 0.1 & 1.2 \\
\hline $\mathbf{Y b}$ & 0.9 & 0.8 & 0.3 & 1.7 & 0.6 & 0.8 & 0.7 & 0.6 & 0.8 & 0.1 & 7.3 \\
\hline Lu & 0.1 & 0.1 & 0.1 & 0.3 & 0.1 & 0.1 & 0.1 & 0.1 & 0.1 & 0.0 & 1.1 \\
\hline Hf & 0.9 & 3.9 & 2.0 & 6.3 & 3.7 & 4.1 & 4.0 & 4.1 & 4.2 & 0.3 & 33.5 \\
\hline$\Sigma$ REE & 584.1 & 134.2 & 46.2 & 163.0 & 149.1 & 170.5 & 139.2 & 105.4 & 169.2 & 18.0 & 1678.9 \\
\hline$\sum$ LREE & 579.4 & 127.7 & 43.3 & 151.5 & 143.2 & 163.8 & 132.8 & 99.2 & 162.4 & 17.2 & 1620.5 \\
\hline$\Sigma$ HREE & 4.7 & 6.5 & 2.9 & 11.5 & 5.9 & 6.7 & 6.3 & 6.2 & 6.8 & 0.8 & 58.4 \\
\hline LREE/HREE & 123.8 & 19.5 & 14.9 & 13.2 & 24.3 & 24.3 & 21.0 & 16.1 & 23.7 & 22.4 & 27.8 \\
\hline
\end{tabular}

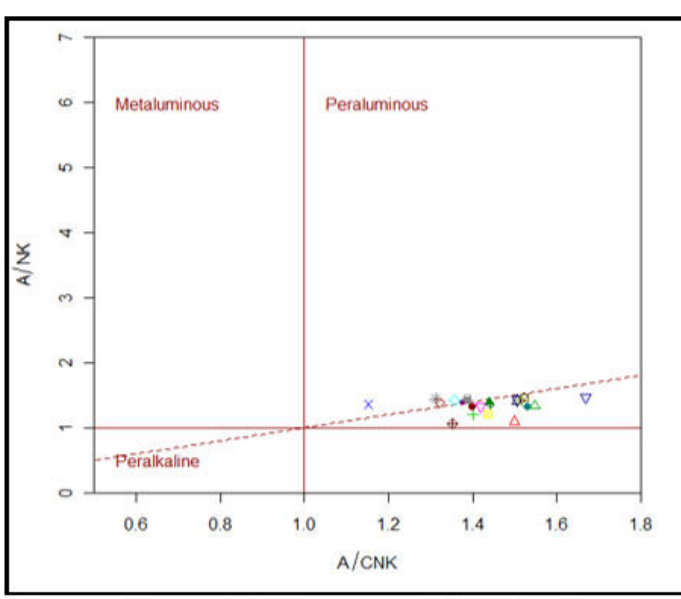

Figure 3: $\mathrm{A} / \mathrm{NK}$ vs. $\mathrm{A} / \mathrm{CNK}$ diagram discriminating metaluminous, peraluminous and peralkaline compositions of the granitoids in the study area after (Shand, 1943).

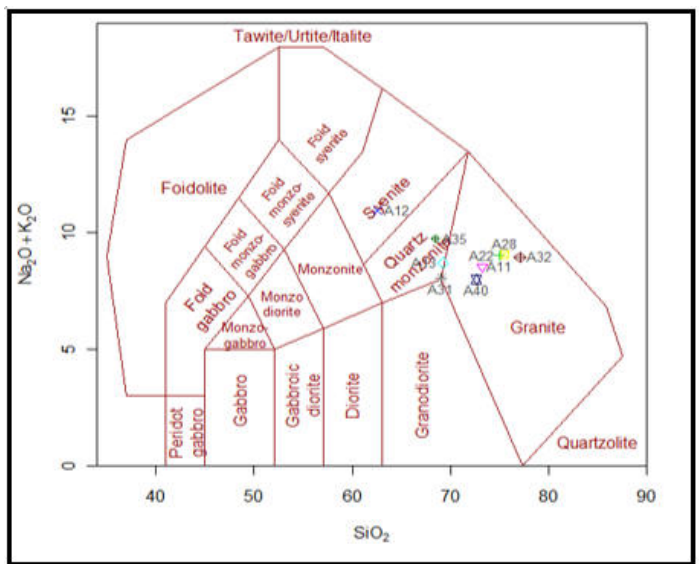

Figure 5: Chemical classfication plot of granitoids after (Middlemost, 1985).

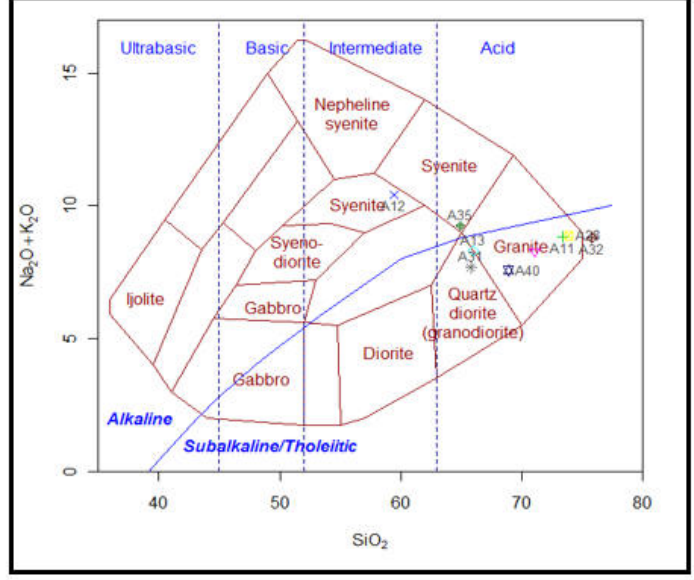

Figure 4: Chemical classfication plot of granitoids after (Cox et al., 1979).

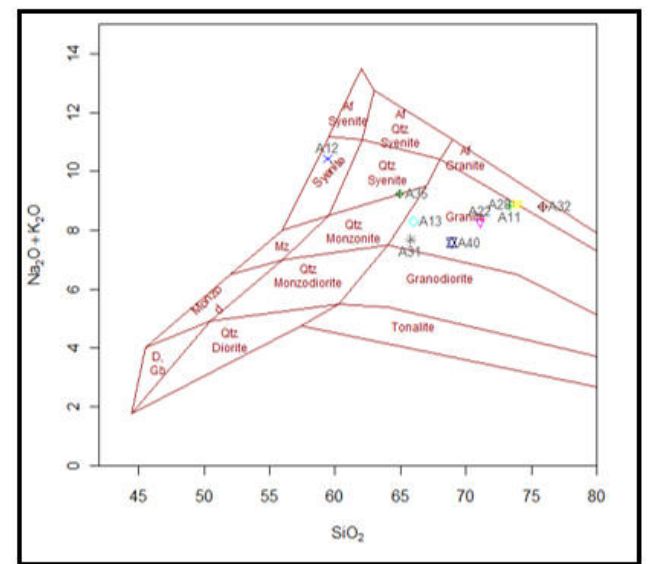

Figure 6: Chemical classfication plot of granitoids after (Middlemost, 1994). 


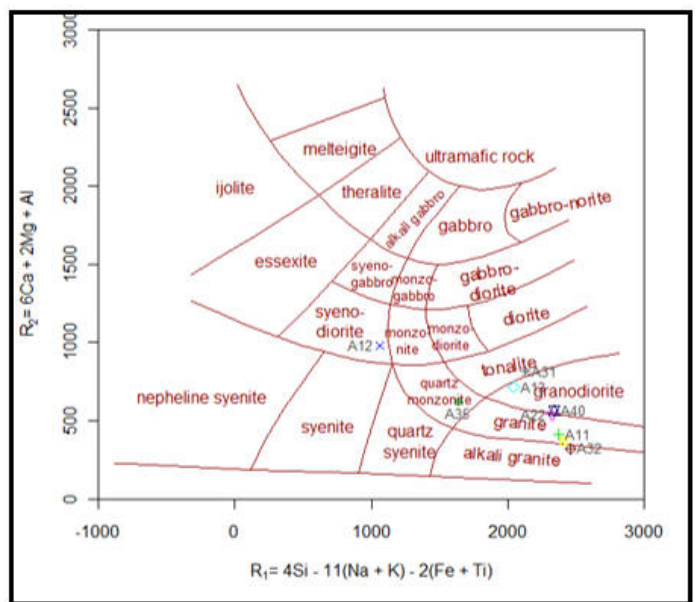

Figure 7: Millication classification proportions plot of granitoids after (De La Roche, et al., 1980)

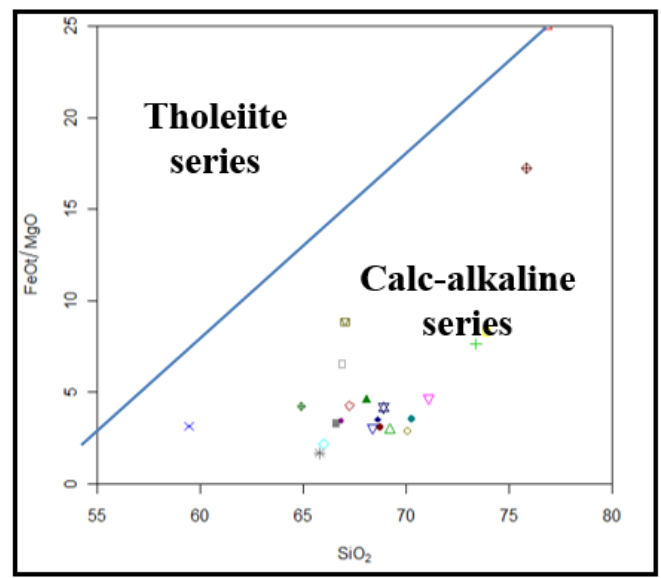

Figure 9: Classification plot of the granitoids in the study area after (Miyashiro, 1974)

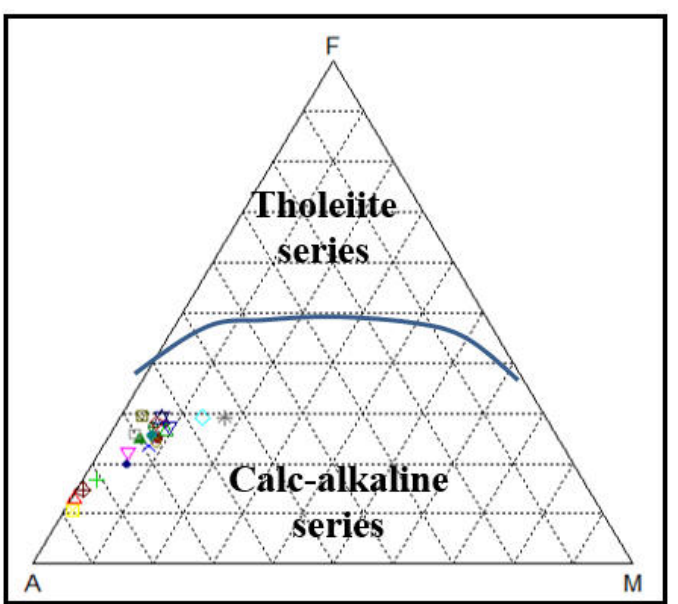

Figure 8: AFM classification plot of the granitoids in the study area after (Irvine and Baragar, 1971)

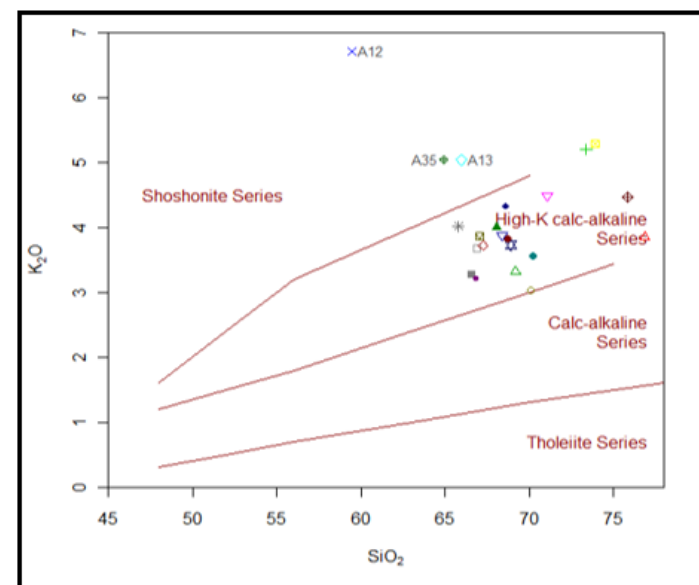

Figure 10: $\mathrm{SiO}_{2}-\mathrm{K}_{2} \mathrm{O}$ classification plot of the granitoids in the study area after (Peccerillo and Taylor ,1976)

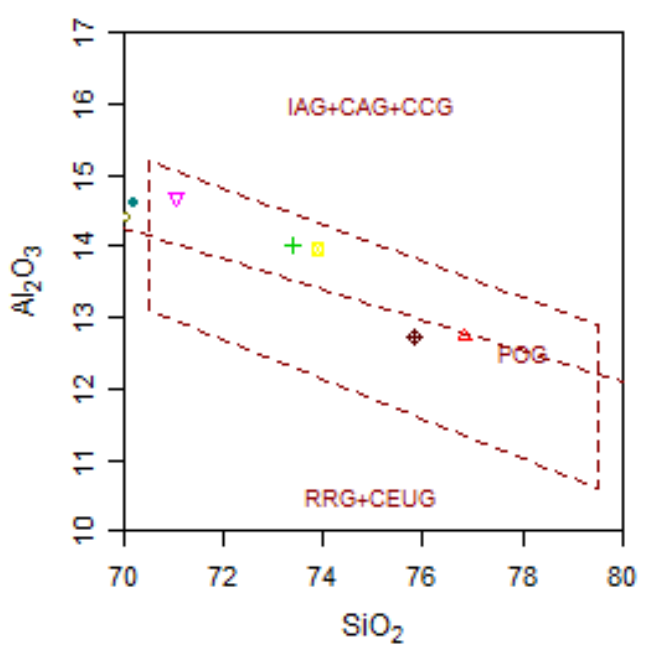

Figure 12: Tectonic discrimination diagram of granitoids in the study area after (Maniar and Piccoli, 1989) 

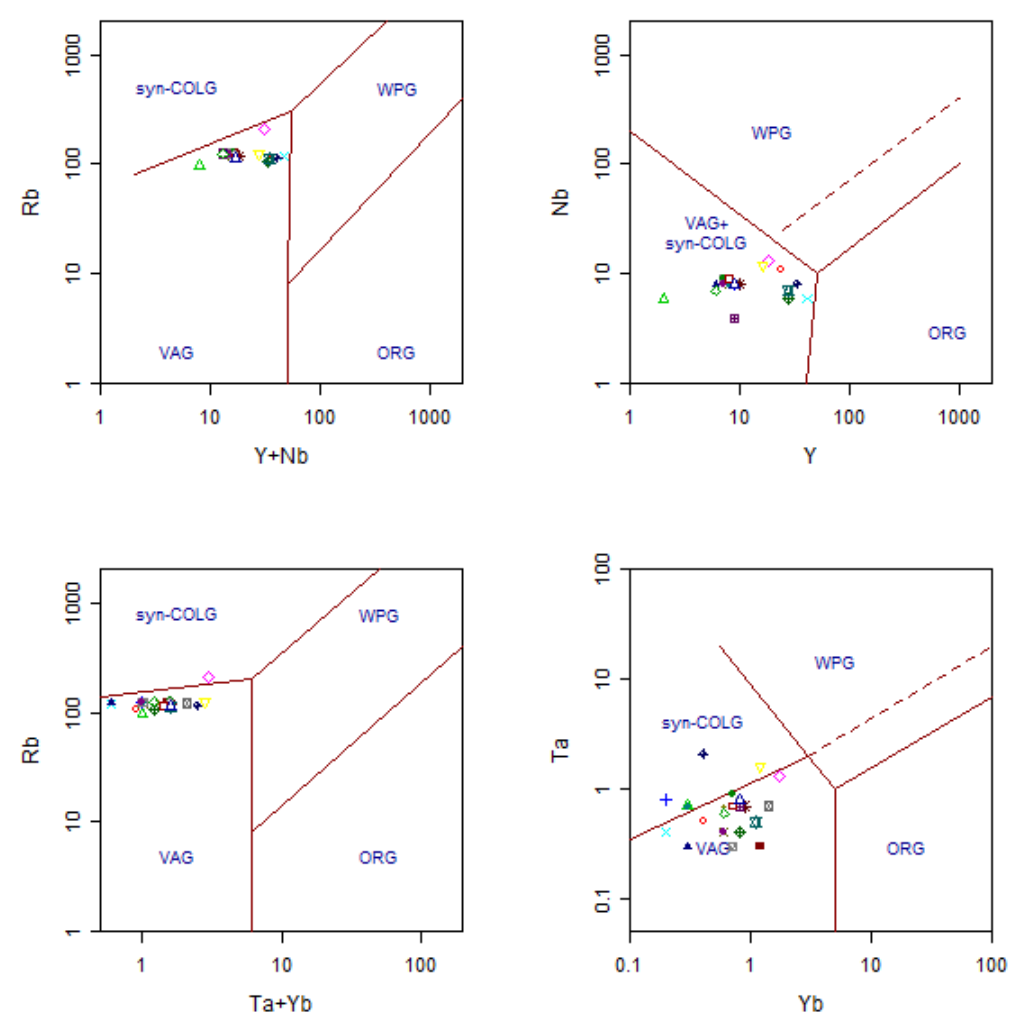

Figure 13: Tectonic discrimination diagram of granitoids in the study area after (Pearce, et. al., 1984)

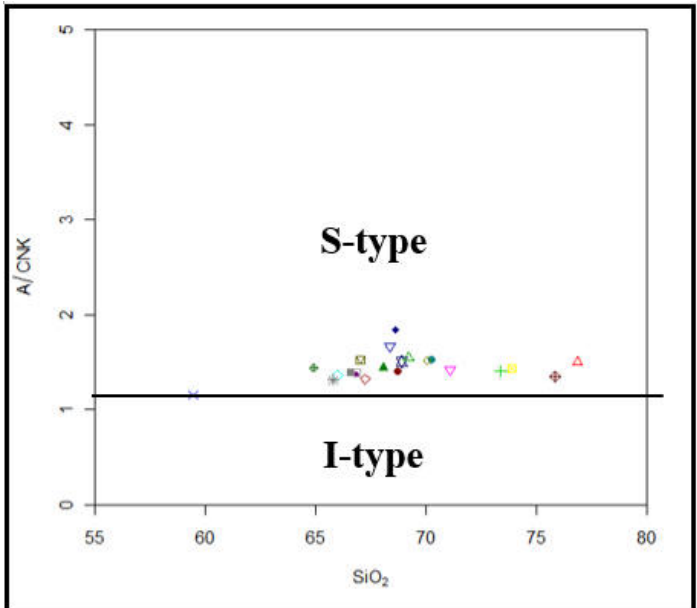

Figure 11: Binary plot for distinction of S-type granitoids in the study area from the I-type after (Chappell and White, 1974; 1992).

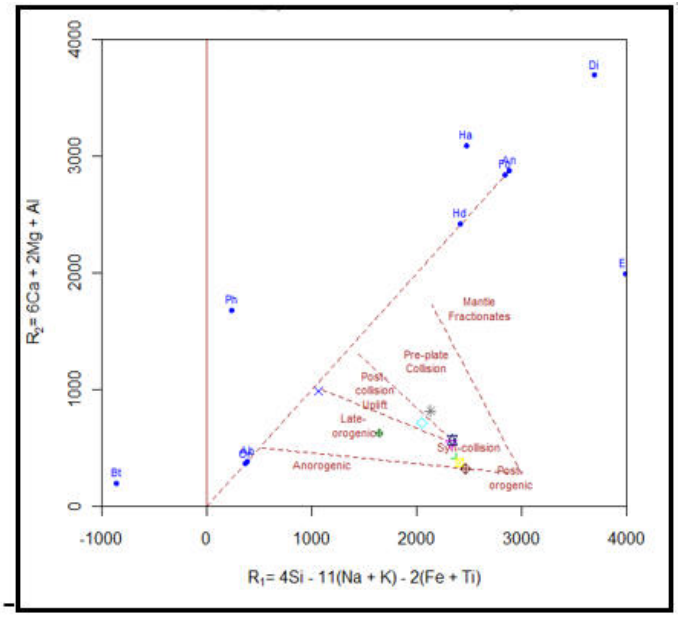

Figure 14: Tectonic discrimination diagram of granitoids in the study area after (Batchelor and Bowden, 1985) 


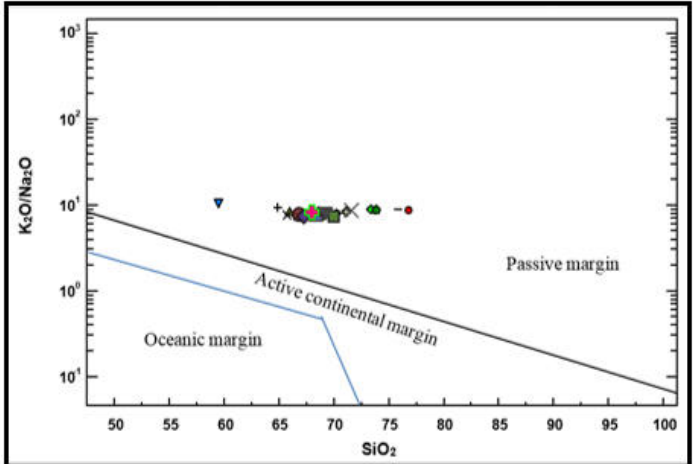

Figure 15: Tectonic discrimination plot of granitoids in the study area after (Roser and Korsch, 1986).

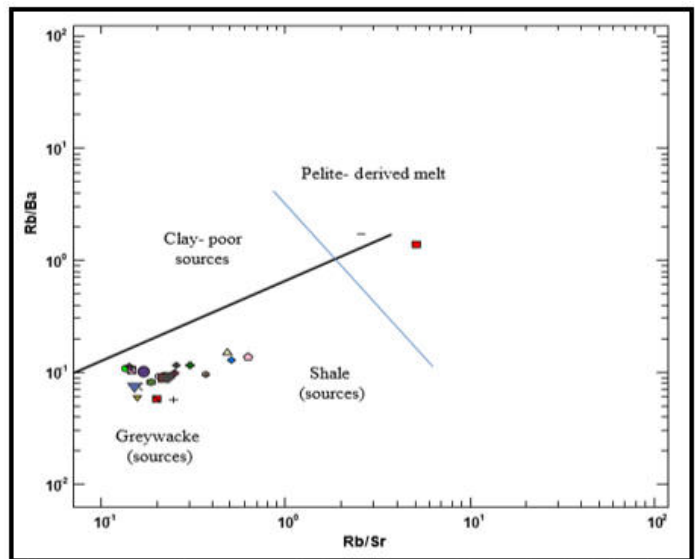

Figure $17: \mathrm{Rb} / \mathrm{Sr}$ vs. $\mathrm{Rb} / \mathrm{Ba}$ plots of granitoids in the study area. Field source rocks are after Sylvester (1998) and Dahlquist, et al. (2007).

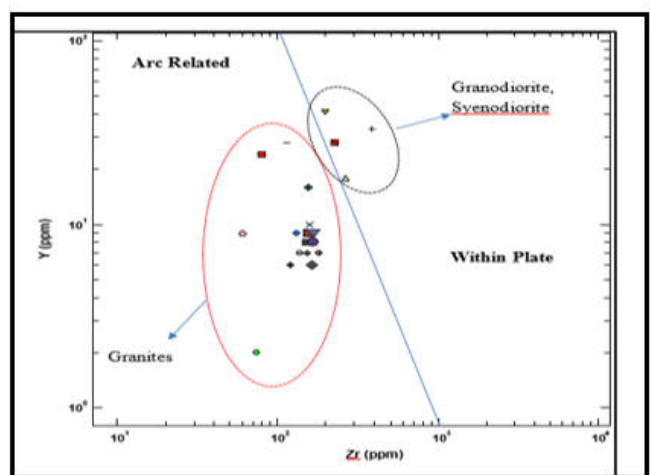

Figure 16: $\mathrm{Zr}$ vs. $\mathrm{Y}$ biaxial geochemical discrimination diagram after (Muller, et al., 2001).

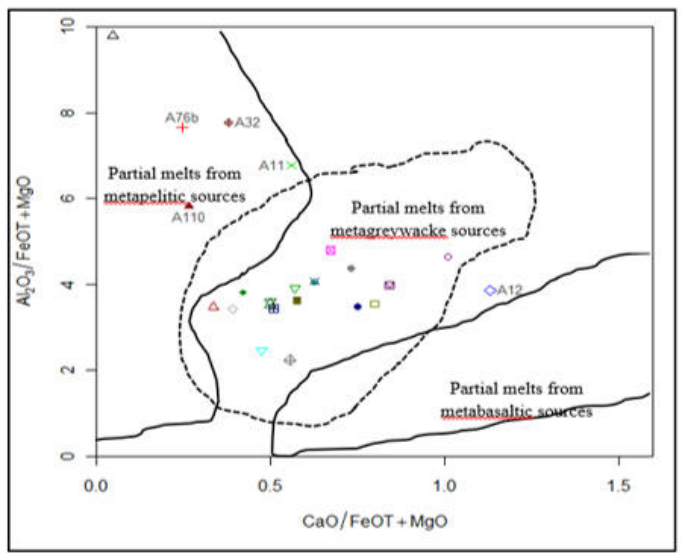

Figure 18: Molar $\mathrm{CaO} / \mathrm{FeOt}+\mathrm{MgO}$ vs. $\mathrm{Al} 2 \mathrm{O} 3 / \mathrm{FeOt}$ $+\mathrm{MgO}$ of granitoids in the study area after (Altherr, et al., 2000)

\section{Discussion}

Tectonic environments of granitoids as categorized by (Maniar and Piccoli, 1989) are 1. island arc granitoids (IAG), 2. continental arc granitoids (CAG), 3. Continental collision granitoids (CCG), 4. Postorogenic granitoids (POG), 5. rift-related granitoids (RRG), 6. Continental epeirogenic uplift granitoids (CEUG) and 7. Oceanic plagiogranites (OP). Of these, IAG, CAG, CCG and POG are considered orogenic granitoids while RRG, CEUG and OP are considered anorogenic granitoids. In this respect granitoids in the study area plots within the orogenic class. The formation of the granitoids with the use of trace element discrimination diagrams as a means of fingerprinting the tectonic environments were also considered and the granitoids plotted in the syn-collision volcanic arc granite field after (Pearce et. al., 1984) which is consistent with the tectonomagmatic setting. Tectonic diagram after (Batchelor and Bowden, 1985) revealed that granites within the study area ranges from syn-collision to late orogenic field. The granites which plotted in the syn-collision field is a characteristic of chemical affinity of syn-collision peraluminous granites with highly fractionated volcanic-arc magmas (Harris et al., 1986). Roser and Korsch (1986) discrimination plot for the granitoids in the study area revealed that they are within passive margin. Meanwhile, biaxial (Zr vs. Y) geochemical discrimination diagram after (Muller, et al., 2001) is an indication of a subduction arc-related features for the granites and within plate source for the granodiorite and syeno-diorite specifically. The petrogenesis of the S-type granitoids in the study area are greywacke sourced from 
the plot of $\mathrm{Rb} / \mathrm{Sr}$ vs. $\mathrm{Rb} / \mathrm{Ba}$ after (Dahlquist et. al., 2007) and Molar $\mathrm{CaO} / \mathrm{FeOt}+\mathrm{MgO}$ vs. $\mathrm{Al}_{2} \mathrm{O}_{3} / \mathrm{FeOt}+\mathrm{MgO}$ after (Altherr et al., 2000) further confirmed that the greywacke is largely metagreywacke and metapelitic sourced during partial melting.

\section{Conclusion}

The granitoids within the study area are peraluminous, ranges from granites, granodiorite, syenite, syeno-diorite and tonalite in places. The granites are calc-alkaline to high calc-alkaline but the syenites relatively high in potassium and depleted in silica plotted in the field of shoshonite series. Recently, (Muller and Groves, 2019) confirmed that shoshonitic and high potassium calc-alkaline magmatism is associated with world class hydrothermal gold-copper mineralization. The tectonic environments of the study area as evidence from geochemical studies revealed that the granitoids are S-type source which is formed during syn-collision to late orogenic field. Apparently of a low temperature origin. The petrogenesis of the S-type granitoids that formed the sub-surface geology of the study area are greywacke which are largely metagreywacke and metapelitic sourced during partial melting.

\section{References}

Ajibade, A. C. (1980). Geotectonic evolution of the Zungeru Region, Nigeria.Unpublished Ph.D. Thesis, University of Wales, Aberystwyth.

Altherr, R., Holl, A., Hegner, E., Langer, C. and Kreuzer, H. (2000). High potassium, calc-alkaline I-type plutonism in the European Variscides, northern Vosges (France) and northern Schwarzwald (Germany), Lithos, (50), p.51-73.

Batchelor, R. A. and Bowden, P. (1985). Petrogenetic interpretation of granitoid rock series using multicationic parameters. Chemical Geology, (48), p.43-55.

Bowden, P. and Turner, D. C. (1974). Peralkaline and associated ring - complexes in the Nigeria - Nigeria Province, West Africa. In: Sǿrensen, H. (ed.). The Alkaline Rocks. New York: John Wiley, p.330 -351.

Chappell, B. W. and White, A. J. R. (1992). I- and S-type granites in the Lachlan Fold Belt. Trans. Royal. Soc. Edin-burgh. 83, 1-26. Also Geological Society of America, (111), p.1-26.

Chappell, B. W. (1996). Magma mixing and the production of compositional variation within granite suites: evidence from the granites of southeastern Australia. Journal of Petrology (37), p. 449-470.

Cox, K. J., Bell, J. D. and Pankhurst, R. J. (1979). The interpretation of igneous rocks, Allen and Unwin, London, p. 450 .

Dada, S.S. (2006). Proterozoic evolution of Nigeria. In: Oshi O (ed.). The basement complex of Nigeria and its mineral resources (A Tribute to Prof. Rahaman, M. A. O.), Akin Jinad and Company, Ibadan, p.29-44.

Dahlquist, J. A., Galindo C., Pankhurst, R. J., Rapela, C. W., Alasino, P. H., Saavedra J. and Fanning, C. M. (2007). Magmatic evolution of the Peñón Rosado granite: Petrogenesis of garnet-bearing granitoids, Lithos, (95), p.177-207.

De La Roche, H., Leterrier, J., Grandclaude, P. and Marchal, M. (1980). A classification of volcanic and plutonic rocks using R1R2-diagram and major element analyses - its relationships with current nomenclature. Chemical Geology, (29), p.183-210. 
Egbuniwe, I. G. (1982). Geotectonic evolution of the Maru Belt, NW Nigeria. Unpublished Ph.D. Thesis, University of Wales, Aberystwyth.

Emmermann R, Daieva L, Schneider J (1975) Petrologic significance of rare earths distribution in granites. Contributions to mineralogy and petrology 52: 267-283.

Fitches, W. R., Ajibade, A. C., Egbuniwe, I. G., Holt, R.W. and Wright, J. B. (1985). Late Proterozoic schist belts and plutonism in NW Nigeria, Journal of the Geological Society of London, (142), p.319-337.

Harris, N. B. W., Pearce, J. A. and Tindle, A. G. (1986). Geochemical characteristics of collision-zone magmatism. In: Coward, M. P. and Ries, A.C. (ed.), Collision tectonics, geological society special publication, (19), p. 67-81.

Holt, R. (1982). Geotectonic evolution of the Anka schist belt in the Precambrian basement complex of northwestern Nigeria. Unpublished Ph.D. Thesis, Open University, England, p.254.

Irvine, T. N. and Baragar, W. R. A. (1971). A guide to the chemical classification of the common volcanic rocks. Canadian Journal of Earth Sciences, (8), p.523-548.

Jones, H. A. and Hockey, R. D. (1964). The geology of part of south-western Nigeria. Geological Survey of Nigeria, Bulletin, (31), p.101.

Maniar, P. D. and Piccoli, P. M. (1989). Tectonic discriminations of granitoids. Geological Society of America, Bulletin (101), p.635-643.

McCurry, P. (1971). Pan-African Orogeny in Northern Nigeria. Geological Society of American Bulletin (82), p.3251-3263.

McCurry, P. (1973). Geology of degree sheet 21, Zaria, Nigeria. Overseas Geology and Mineral Resources (45), p. $1-30$.

Middlemost, E. A. K. (1985). Magmas and magmatic rocks. An introduction to igneous petrology. Longman group limited, London, New York, p. 266.

Middlemost, E. A. K. (1994). Naming materials in the magma/igneous rock system, earth science reviews, Elsevier, (37), p. 215-224.

Miyashiro, A. (1974). Volcanic rock series in island arcs and active continental margins. American Journal of Sciences, (274), p.321-355.

Müller, D., Franz, L., Herzig, P. M. and Hunt, S. (2001). Potassic igneous rocks from the vicinity of epithermal gold mineralization, Lihir Island, Papua New Guinea. Lithos (75), p. 163-186.

Muller, D. and Groves, D. I. (2019). Potassic igneous rocks and associated gold-copper mineralization (5 $5^{\text {th }}$ ed.). Mineral resources review. Springer-Verlag Heidelberg, p.398.

Obiora SC, Ukaegbu VU (2010) Preliminary investigation of the petrogenesis and geotectonic setting of the Precambrian Basement Complex rocks around northcentral Nigeria using trace and rare-earth elements geochemistry. Journal Mining Geology 46: 127-137.

Pearce, J. A., Harris, N. B. W. and Tindle, A. G. (1984). Trace element discrimination diagrams for the tectonic interpretation of granitic rocks. Journal of Petrology, (25), p.956-983.

Peccerillo, A. and Taylor, S. R. (1976). Geochemistry of Eocene calc-alkaline rocks from Kastamonu area, northern Turkey. Contributions to mineralogy and petrology. (58), p. 63-81.

Rahaman, M. A. (1976). Review of the basement geology of south-western Nigeria. In: Kogbe, C. (ed.), Geology 
of Nigeria, $2^{\text {nd }}$ edition, Elizabethan Publishers, Lagos, p.41-58.

Rahaman, M. A. (1988). Recent advances in study of the basement complex of Nigeria Precambrian geology of Nigeria. In: Oluyide, P.O. et al., (ed.), Precambrian geology of Nigeria. Geological survey of Nigeria publication, Kaduna, p.11-43.

Roser, B. P. and Korsch, R. J. (1988). Provenance signature of sandstone-mudstone suites determined using discriminant function analysis of major element data. Chemical Geology, (67), p.119-139.

Shand, S. J. (1943). The eruptive rocks: 2nd edition, John Wiley, New York, p.444.

Turner, D. C. (1983). Upper Proterozoic schist belts in the Nigerian sector of the Pan-African Province of West Africa. Precambrian Resources, (21), p.55-79.

Woakes, M., Rahaman, M. A. and Ajibade, A. C. (1987). Some metallogenetic features of the Nigerian basement. Journal of African Earth Sciences, (6), p.54-64.

Wright, J.B., (1985). Geology and mineral resources of West Africa. George Allen and Unwin, London, 187pp. 